\title{
SOME RANDOM FIXED POINT THEOREMS FOR CONDENSING OPERATORS
}

\author{
V. M. SEHGAL AND CHARLES WATERS
}

\begin{abstract}
In this paper we obtain several random fixed point theorems including a stochastic generalization of the classical Rothe fixed point theorem. The results herein improve a recent result of Bharucha-Reid and Mukherjea and also some similar results of Itoh.
\end{abstract}

1. Throughout, let $(\Omega, \Sigma)$ be a measurable space $(\Sigma=$ sigma algebra) and $X$ a nonempty subset of a Banach space $E$. In a recent paper [1], Bharucha-Reid and Mukherjea (see also Mukherjea [6]) have given sufficient conditions for a stochastic analogue of Schauder's fixed point theorem for a random operator $T: \Omega \times X \rightarrow X$. Itoh [5] introduced random condensing operators and considerably improved their result (see Lemma 1 below). In this paper, we consider random operators $T: \Omega \times X$ $\rightarrow E$ and give sufficient conditions for the existence of a measurable map $\phi: \Omega \rightarrow X$ satisfying a Browder-Fan [4] type result. As a consequence, a stochastic generalization of the well-known Rothe fixed point theorem is obtained. A random analogue of the Krasnoselskii fixed point theorem for the sum of two operators is given for a Hilbert space.

2. Definitions. For most definitions we refer to Bharucha-Reid [2]. A mapping $\phi: \Omega \rightarrow E$ is measurable iff $\phi^{-1}(U) \in \Sigma$ for each open subset $U$ of $E$. The mapping $T: \Omega \times X \rightarrow E$ is a random operator iff for each fixed $x \in X$, the mapping $T(\cdot, x): \Omega \rightarrow E$ is measurable, and it is continuous if for each $\omega \in \Omega$, the mapping $T(\omega, \cdot): X \rightarrow E$ is continuous. A measurable mapping $\phi: \Omega \rightarrow E$ is a random fixed point of the random operator $T: \Omega \times X \rightarrow E$ iff $T(\omega, \phi(\omega))=\phi(\omega)$ for each $\omega \in \Omega$.

For a subset $A$ of $E$, let $\delta(A)$ denote the diameter, $\operatorname{int}(A)$ the interior, $\operatorname{co}(A)$ the convex hull, $\partial(A)$ the boundary and $\gamma(A)$ the measure of noncompactness of the set $A$. Note that $\gamma(A)=\inf \{\varepsilon>0: A$ can be covered by a finite number of subsets $A_{1}, A_{2}, \ldots, A_{n}$ of $E$ with $\delta\left(A_{i}\right) \leqslant \varepsilon$ for each $\left.i\right\}$. It is easy to show that (i) $\gamma(A)=0$ iff $A$ is totally bounded and $\gamma(A+B)=\gamma(A)$ if $B$ is totally bounded, (ii) $\gamma(A) \leqslant \gamma(B)$ if $A \subseteq B$ with $\gamma(\operatorname{co}(A))=\gamma(A)$. A random operator $T: \Omega \times X \rightarrow E$ is called (a) condensing if for each bounded subset $A \subseteq X$ with $\gamma(A)>0, \gamma(T(\omega, A))<\gamma(A)$ for each fixed $\omega \in \Omega$; (b) contraction if there exists a mapping $\alpha: \Omega \rightarrow[0,1)$ satisfying

Received by the editors August 5, 1982.

1980 Mathematics Subject Classification. Primary 60H25, 47H10.

Key words and phrases. Measurable maps, random fixed point, condensing random operator.

(C)1984 American Mathematical Society $0002-9939 / 84 \$ 1.00+\$ .25$ per page 
$\|T(\omega, x)-T(\omega, y)\| \leqslant \alpha(\omega)\|x-y\|$ for each $x, y \in X, \omega \in \Omega ;$ (c) compact (bounded) iff for each fixed $\omega \in \Omega$, the set $T(\omega, X)$ is totally bounded (bounded) subset of $E$.

Recall that if $U$ is a convex neighborhood of the origin in $E$, then the Minkowski function $p$ of $U$ is defined on $E$ by $p(x)=\inf \{\varepsilon>0: x \in \varepsilon U\}$. It is continuous, subadditive, positively homogeneous and satisfies $\{x: p(x)<1\} \subseteq U \subseteq\{x: p(x) \leqslant$ 1) $\subseteq$ closure of $G$.

The result below is due to Itoh [5, Theorem 2.1] and is basic to our main result.

Lemma 1. Let $X$ be a separable closed and convex subset of a Banach space $E$ and $T: \Omega \times X \rightarrow X$ be a bounded, continuous condensing random operator. Then $T$ has $a$ random fixed point.

3. The following is a Browder-Fan [4] type result.

TheOREM 1. Let $X$ be a separable, closed and convex subset of a Banach space $E$ with $\operatorname{int}(X) \neq \varnothing$ and let $T: \Omega \times X \rightarrow E$ be a bounded, continuous condensing random operator. Then for each $y \in \operatorname{int}(X)$, there exists a measurable map $\phi=\phi(y): \Omega \rightarrow X$ satisfying for each $\omega \in \Omega$,

$$
p(T(\omega, \phi(\omega))-\phi(\omega))=\min \{p(T(\omega, \phi(\omega))-x): x \in X\},
$$

where $p=p(y)$ is the Minkowski function of $(X-y)$. Further, if

$$
\text { . } p(T(\omega, \phi(\omega))-y) \leqslant 1 \text { for some } \omega \in \Omega,
$$

then $T(\omega, \phi(\omega))=\phi(\omega)$.

As a consequence of Theorem 1, we have the following stochastic generalization of the well-known Rothe fixed point theorem. Note that for any closed $X$ of $E$ if $x \in \operatorname{int}(X)$ and $y \in E \backslash X$, then there exists [1, Lemma 1] $a, c$ with $0<c<1$ such that $c x+(1-c) y \in \partial X$.

THEOREM 2. Let $X$ be a separable, closed and convex subset of a Banach space $E$ and $T: \Omega \times X \rightarrow E$ be a bounded, continuous condensing random operator. If $T(\Omega \times \partial X)$ $\subseteq X$, then $T$ has a random fixed point

Proof. If $X=\partial X$, then by Lemma $1, T$ has a random fixed point. If $\operatorname{int}(X) \neq \varnothing$, then for any $y \in \operatorname{int}(X)$, it follows by Theorem 1 that there is a measurable map $\phi=\phi(y): \Omega \rightarrow X$ satisfying (1). To show $\phi$ is in fact a random fixed point, it suffices to show that $p(T(\omega, \phi(\omega))-y) \leqslant 1$ for each $\omega \in \Omega$. Suppose for some $\omega \in \Omega, p(T(\omega, \phi(\omega))-y)>1$. Then $T(\omega, \phi(\omega)) \notin X$. This implies by hypothesis that $\phi(\omega) \in \operatorname{int}(X)$. Consequently, there is a $c$ with $0<c<1$, such that $z=c \phi(\omega)$ $+(1-c) T(\omega, \phi(\omega)) \in \partial X$. Hence by $(1)$,

$$
p(T(\omega, \phi(\omega))-\phi(\omega)) \leqslant p(T(\omega, \phi(\omega))-z)=c p(T(\omega, \phi(\omega))-\phi(\omega)) .
$$

This yields $p(T(\omega, \phi(\omega))-\phi(\omega))=0$. This implies that $p(T(\omega, \phi(\omega))-y) \leqslant$ $p(\phi(\omega)-y) \leqslant 1$, which contradicts the assumption.

It may be remarked that since a compact random operator is bounded and condensing, Theorem 10 in [1] is a special case of Theorem 2.

The lemma below simplifies the proof of Theorem 1 . 
Lemma 2. Let $f: \Omega \rightarrow E$ and $l: \Omega \rightarrow[0,1]$ be measurable mappings. Then for any fixed $y \in E$, the mapping $h: \Omega \rightarrow E$ defined by $h(\omega)=l(\omega) f(\omega)+(1-l(\omega)) y$ is measurable.

Proof. We first show that $k(\omega)=l(\omega) \cdot f(\omega)$ is measurable. To prove this, choose a sequence $\left\{S_{n}\right\}$ of simple functions, $S_{n}: \Omega \rightarrow[0,1]$ such that $S_{n} \rightarrow l$ uniformly. Let $S_{n}=\sum_{i=1}^{m(n)} \alpha_{i n} \chi_{E_{i n}}$ where $E_{i n} \cap E_{j n}=\varnothing$ if $i \neq J, \cup_{i=1}^{m(n)} E_{i n}=\Omega, E_{i n} \in \Sigma$ and $\alpha_{i n} \in[0,1]$ for each $i$. Define a sequence $g_{n}: \Omega \rightarrow E$ by $g_{n}(\omega)=S_{n}(\omega) \cdot f(\omega)$. Then for each open set $U$ in $E$

$$
g_{n}^{-1}(U)=\bigcup_{i=1}^{m(n)} \begin{cases}E_{i n} \cap f^{-1}\left(\alpha_{i n}^{-1} U\right) & \text { if } \alpha_{i n} \neq 0, \\ E_{i n} & \text { if } \alpha_{i n}=0 \text { and } \theta \in U, \\ \phi & \text { if } \alpha_{i n}=0 \text { and } \theta \notin U .\end{cases}
$$

Thus, $g_{n}$ is measurable for each $n$. Since $g_{n} \rightarrow k$, it follows that $k$ is measurable. Now, since $h(\omega)=l(\omega)(f(\omega)-y)+y$ and $f+u$ is measurable for each $u \in E$, it follows that $h$ is measurable.

Proof of Theorem 1. Let $y$ and $p$ be as in Theorem 1. Define a mapping $l: \Omega \times X \rightarrow[0,1]$ by

$$
l(\omega, x)=\max \{1, p(T(\omega, x)-y)\}^{-1} .
$$

Then $l$ is a continuous random operator and $l(\omega, x) p(T(\omega, x)-y) \leqslant 1$ for each $(\omega, x)$. Define $g: \Omega \times X \rightarrow E$ by

$$
g(\omega, x)=l(\omega, x) T(\omega, x)+(1-l(\omega, x)) y .
$$

We show that $g$ satisfies the conditions of Lemma 1. Clearly by hypothesis and (3), $g$ is a bounded, continuous random operator and since $p(g(\omega, x)-y)=$ $l(\omega, x) p(T(\omega, x)-y) \leqslant 1$, it follows that $g(\Omega \times X) \subseteq X$. Further, for any bounded set $B$ of $X$ with $\gamma(B)>0$ and $\omega \in \Omega$, it follows by (3) that

$$
\gamma(g(\omega, B)) \leqslant \gamma(\operatorname{co}(T(\omega, x) \cup y))=\gamma(T(\omega, B)) \leqslant \gamma(B) .
$$

Thus, $g$ is condensing. Consequently, by Lemma 1 , there exists a measurable map $\phi: \Omega \rightarrow X$ with $g(\omega, \phi(\omega))=\phi(\omega)$ for each $\omega \in \Omega$. This implies that for each $\omega \in \Omega$

$$
l(\omega, \phi(\omega)) T(\omega, \phi(\omega))+(1-l(\omega, \phi(\omega))) y=\phi(\omega) .
$$

Now for any $\omega \in \Omega$, either (a) $p(T(\omega, \phi(\omega))-y) \leqslant 1$ or (b) $p(T(\omega, \phi(\omega))-y)>1$. In case of (a), it follows by (2) $l(\omega, \phi(\omega))=1$ and hence by (4) $T(\omega, \phi(\omega))=\phi(\omega)$. If (b) holds then by (2) $l(\omega, \phi(\omega)) p(T(\omega, \phi(\omega))-y)=1$ and since for any $x \in X$, $p(x-y) \leqslant 1$, it follows by (4) that for any $x \in X$,

$$
p(T(\omega, \phi(\omega))-\phi(\omega))=p(T(\omega, \phi(\omega))-y)-1 \leqslant p(T(\omega, \phi(\omega))-x) .
$$

Since $\phi(\omega) \in X$, the last inequality implies that $p(T(\omega, \phi(\omega))-\phi(\omega))=$ $\min \{p(T(\omega, \phi(\omega))-x): x \in X\}$. Thus (a) and (b) provide the conclusions of Theorem 1.

If $X$ is a closed and convex subset of a Hilbert space $H$, then it is well known (see Cheney and Goldstein [3]) that there exists a metric projection $P: H \rightarrow X$ such that 
$P$ is nonexpansive $(\|P x-P y\| \leqslant\|x-y\|, x, y \in H)$ and satisfies $\|P x-x\|=$ $\min \{\|z-x\|: z \in X\}$ for each $x \in H$. Using the metric projection, an interesting and also a stronger random version (see [5, Theorem 2.4] or [1, Theorem 12]) of Krasnoselskii's fixed point theorem can be obtained in $H$. In fact, we have

THEOREM 3. Let $X$ be a separable closed and convex subset of a Hilbert space $H$ and $A, B: \Omega \times X \rightarrow H$ be random operators such that $A$ is a contraction and $B$ is compact and continuous. Then there exists a measurable map $\phi: \Omega \rightarrow X$ such that for each $\omega \in \Omega$,

$$
\|T(\omega, \phi(\omega))-\phi(\omega)\|=\min \{\|T(\omega, \phi(\omega))-x\|: x \in X\}
$$

where $T=A+B$. If additionally $T(\Omega \times \partial X) \subseteq X$, then $\phi$ is a random fixed point.

Proof. Let $y$ be a fixed element of $X$. Define $g=g(y): \Omega \times X \rightarrow X$ by $g(\omega, x)=$ $P(A(\omega, x)+B(\omega, y))$, where $P$ is the metric of $H$ onto $X$. Then $g$ is a random contraction operator and hence (see Bharucha-Reid [2, p. 109]) there exists a measurable map $\xi=\xi(y): \Omega \rightarrow X$ satisfying for $\omega \in \Omega, \quad P(A(\omega, \xi(\omega))+B(\omega, y))$ $=\xi(\omega)$. Define $h: \Omega \times X$ by $h(\omega, y)=\xi(y)(\omega),(\omega, y) \in \Omega \times X$. Then $h$ is a random operator and satisfies

$$
P(A(\omega, h(\omega, y))+B(\omega, y))=h(\omega, y)
$$

for each $(\omega, y) \in \Omega \times X$. It follows by (5) that for $\omega \in \Omega, x, y \in X, \| h(\omega, x)-$ $h(\omega, y)\left\|\leqslant(1-\alpha(\omega))^{-1}\right\| B(\omega, x)-B(\omega, y) \|$, where $\alpha(\omega)$ is the contraction constant (see definition). This implies that $h$ is bounded and continuous. Further, since $\gamma(B(\omega, X))=0$, it follows by (5) that $\gamma(h(\omega, X)) \leqslant \gamma(A(\omega, h(\omega, X))) \leqslant$ $\alpha(\omega) \gamma(h(\omega, X))$ and consequently $\gamma(h(\omega, X))=0$ for each $\omega \in \Omega$. This implies that $h$ is condensing. Thus by Lemma 1 , there is a measurable map $\phi: \Omega \rightarrow X$ with $h(\omega, \phi(\omega))=\phi(\omega)$ for each $\omega \in \Omega$. Thus, $P(A(\omega, \phi(\omega))+B(\omega, \phi(\omega)))=\phi(\omega)$ and in view of the definition of $P$, we have

$$
\|T(\omega, \phi(\omega))-\phi(\omega)\|=\min \{\|T(\omega, \phi(\omega))-x\|: x \in X\}
$$

The proof of the last part is contained in the proof of Theorem 2. In fact, if for some $\omega \in \Omega, T(\omega, \phi(\omega)) \neq \phi(\omega)$, then by the above conclusion, $\phi(\omega) \in \operatorname{int}(X)$ and $T(\omega, \phi(\omega)) \in H \backslash X$. Consequently, there is a $c, 0<c<1$ with

$$
(1-c)\|T(\omega, \phi(\omega))-\phi(\omega)\|=0
$$

which contracts the assumption.

It may be remarked that the random analogue of Krasnoselskii's theorem [5, Theorem 2.4], or [1, Theorem 12] in $E$ requires the additional hypothesis that $A(\omega, x)+B(\omega, y) \in X$ for each $\omega \in \Omega$ and $x, y \in X$. Thus, Theorem 3 in $H$ is an improvement over $E$. 


\section{REFERENCES}

1. A. T. Bharucha-Reid, Fixed point theorems in probabilistic analysis, Bull. Amer. Math. Soc. 82 (1976), $641-645$.

2. Random integral equations, Academic Press, New York, 1972.

3. W. E. Cheney and A. A. Goldstein, Proximity maps for convex sets, Proc. Amer. Math. Soc. 10 (1959), 448-450.

4. Ky Fan, Extensions of two fixed point theorems of F. E. Browder, Math. Z. 112 (1969), 230-240.

5. S. Itoh, Random fixed point theorems with an application to random differential equations in Banach spaces, J. Math. Anal. Appl. 67 (1979), 261-273.

6. A. Mukherjea, Transformations aléatoires séparables, Théorème du point fixe aléatoire, C. R. Acad. Sci. Paris Sér. A-B 263 (1966), 393-395.

7. C. H. Su and V. M. Sehgal, Some fixed point theorems for nonexpansive mappings in locally convex spaces, Boll. Un. Mat. Ital. (4) 10 (1974), 598-601.

Department of Mathematics, University of Wyoming, LaRamie, Wyoming 82071 